\title{
Acknowledgement to Reviewers of Journal of Sensor and Actuator Networks in 2018
}

\author{
Journal of Sensor and Actuator Networks Editorial Office \\ MDPI, St. Alban-Anlage 66, 4052 Basel, Switzerland \\ Published: 15 January 2019
}

\begin{abstract}
Rigorous peer-review is the corner-stone of high-quality academic publishing. The editorial team greatly appreciates the reviewers who contributed their knowledge and expertise to the journal's editorial process over the past 12 months. In 2018, a total of 52 papers were published in the journal, with a median time to first decision of 17 days and a median time to publication of 50 days. The editors would like to express their sincere gratitude to the following reviewers for their cooperation and dedication in 2018:
\end{abstract}

Adnane, Asma

Agiwal, Mamta

Alam, Mushfiqul

Ali, G. G. Md. Nawaz

Amaro, José Pedro

Amhoud, El-Mehdi

Andersson, Karl

Annamdas, Venu GM

Bender, Paul

Benedetto, Francesco

Bernhard, H.-P.

Bezzateev, Sergey V.

Bianchi, Valentina

Blech, Jan Olaf

Bonastre Pina, Alberto Miguel

Boulogeorgos, Alexandros-Apostolos A.

Brauner, Philipp

Brezočnik, Lucija

Brunner, Andreas

Butun, Ismail

Campelo Rivadulla, José Carlos

Canas Ferreira, João

Carvalho, Lidia

Castillejo Parrilla, Pedro

Cerro, Gianni

Chang, Kuo-Jen

Chatzigiannakis, Ioannis

Chen, Chao-Chun

Chen, Chi-Hua

Chen, Sanjian

Cho, Young Im

Cięszczyk, Sławomir

Ciuffoletti, Augusto
Corchado Rodríguez, Juan Manuel

Correia, Sérgio

Cueva, Román Alcides Lara

Cui, Hui

Curiac, Daniel

Dardanelli, Gino

De Castro, Angel

De la Prieta Pintado, Fernando

De Sutter, Bjorn

Dey, Shuvashis

Diez, Luis

Domaneschi, Marco

Domaszewicz, Jarosław

Dulman, Stefan

Eftekhar Azam, Saeed

Elgenaidi, Walid

Fereidountabar, Amirhossein

Fernandes, Telmo

Ferreira, Artur J.

Fidge, Colin

Frasca, Paolo

Frontoni, Emanuele

Menon, Varun G.

Gallo, Francesco

García-Diego, Fernando J.

Garcia-Naya, Jose A.

Garcia-Palacios, Emiliano

Garry, Tony

Gatteschi, Valentina

Goldoni, Emanuele

González Briones, Alfonso

Grochla, Krzysztof

Hakem, Nadir 
He, Zongjian

Heydari, Shahram

Horvath, Tomas

Hsia, Kuo-Hsien

Huang, Chung-Chi

Huang, Tony

Hung, Ho-Lung

Imani, Mahdi

Ionita, Silviu

Jamthe, Anagha

Johanyak, Zsolt Csaba

Kaboli, Mohsen

Kambayashi, Yasushi

Kang, Byungseok

Kantarci, Burak

Kari, Chadi

Karimov, Timur

Karyotis, Vasileios

Khalajmehrabadi, Ali

Khan, Tareq

Kim, Jung Tae

Kim, Sung Suk

Kolomvatsos, Kostas

Koppányi, Zoltán

Kos, Marko

Kravari, Kalliopi

Krejcar, Ondrej

Kuba, Michal

Kulathumani, Vinod

Kuo, Yaw-Wen

Labus, Aleksandra

Lanza-Gutierrez, Jose M.

Lavric, Alexandru

Lee, Brian

Lee, Il-Gu

Lee, Jae Woong

Leister, Wolfgang

Lin, Tsung-Hung

Lin, Wei

Linstead, Erik

Liu, Anfeng

Liu, Chunlei

Liu, Jiechao

Lu, Jiang

Lui, Eric

Ma, Zhou

Mahapatra, Chinmaya

Marszałek, Zbigniew

Maselli, Gaia

Masini, Barbara Mavi

Massin, Raphaël

Mauri, Jaime Lloret
Meisen, Tobias

Mihalache, Sanda Florentina

Milanova, Mariofanna

Minato, Atsushi

Mishra, Amitabh

Moh, Sangman

Morimoto, Naoyuki

Muelas, David

Nadeem, Tamer

Naranjo-Hernández, David

Nguyen, Ngoc Tu

Ochoa-Zezzati, Alberto

Oneto, Luca

Opromolla, Roberto

Orsino, Antonino

O'Shea, Tim

Pagiatakis, Gerasimos

Paredes-Madrid, Leonel

Park, Jaehyun

Parrein, Benoît

Pau, Giovanni

Perazzo, Pericle

Pereira Da Silva, Aloizio

Petrellis, Nikos

Piraino, Francesco

Pomorski, Denis

Prentkovskis, Olegas

Pricop, Emil

Primatesta, Stefano

Qiao, Yuansong

Qiao, Zhijun

Ramos, Victoria

Reis, Catarina I.

Riguidel, Michel

Robles, Ramiro

Roldán Gómez, Juan Jesús

Romero Brufau, Santiago

Samie, Farzad

Sanwar Hosen, A. S. M.

Sawacha, Zimi

Saxena, Navrati

Schatz, Bruce R.

Schmid, Giovanni

Schmidtke, Hedda

Schoeneich, Radosław

Selvaprabhu, Poongundran

Sheng, Duo

Sheu, Jeng-Shin

Shojafar, Mohammad

Shokrolah Shirazi, Mohammad

Singh, Keshav

Sisinni, Emiliano 
Sood, Keshav

Sperlì, Giancarlo

Stroulia, Eleni

Suarez, Alvaro

Suciu, George

Takabayashi, Kento

Tervonen, Jouni K.

Thornton, Douglas

Tkach, Itshak

Torres, Pedro M. B.

Tran, Gia Khanh

Trevathan, Jarrod

Triviño, Alicia

Trotta, Angelo

Tsai, Ming-Fong

Tsai, Pei-Wei

Tseng, Shu-Ming

Tsiigkaridis, John (Ioannis)

Tsinos, Christos

Tsiropoulou, Eirini Eleni

Turgut, Damla

Tutueva, Aleksandra
Uslar, Mathias

Valera, Enrique

Valtierra-Rodriguez, Martin

Vasilateanu, Andrei

Venčkauskas, Algimantas

Verticale, Giacomo

Vladyko, Andrei

Wang, Jun

Weitzen, Jay A.

Wilkins, Ross

$\mathrm{Xu}, \mathrm{He}$

Yan, Zheng

Yelamarthi, Kumar

Yeo, Joo Chuan

Yin, Zhendong

Zagan, Ionel

Zappatore, Marco

Zhang, Dongsheng

Zhang, XinMing

Zhao, Jun

Zhao, Yubin

Zolotová, Iveta

(C) 2019 by the author. Licensee MDPI, Basel, Switzerland. This article is an open access article distributed under the terms and conditions of the Creative Commons Attribution (CC BY) license (http://creativecommons.org/licenses/by/4.0/). 